\title{
Le temps des élites en Gaule atlantique
}

José Gomez de Soto

\section{OpenEdition}

\section{Journals}

Édition électronique

URL : http://journals.openedition.org/rao/2228

DOI : $10.4000 /$ rao.2228

ISBN : 978-2-7535-3432-2

ISSN : 1775-3732

Éditeur

Presses universitaires de Rennes

Édition imprimée

Date de publication : 25 décembre 2013

Pagination : 315-316

ISBN : 978-2-7535-3430-8

ISSN : 0767-709X

\section{Référence électronique}

José Gomez de Soto, "Le temps des élites en Gaule atlantique », Revue archéologique de l'Ouest [En ligne], 30 | 2013, mis en ligne le 25 décembre 2013, consulté le 06 décembre 2020. URL : http:// journals.openedition.org/rao/2228; DOI : https://doi.org/10.4000/rao.2228

Ce document a été généré automatiquement le 6 décembre 2020.

Tous droits réservés 


\title{
Le temps des élites en Gaule atlantique
}

\author{
José Gomez de Soto
}

\section{RÉFÉRENCE}

Milcent P.-Y., 2012 - Le temps des élites en Gaule atlantique. Chronologie des mobiliers et rythmes de constitution des dépôts métalliques dans le contexte européen (XIII ${ }^{e}-\mathrm{VII}{ }^{e}$ s. av.J.-C.), Rennes, PUR, coll. « Archéologie \& Culture », 253 p., 83 pl. (ISBN 978-2-7535-1812-4, $26 €)$

1 Après Le premier âge du Fer en France centrale (Paris, Société préhistorique française, 2004) publication de sa thèse de doctorat, et un nombre appréciable d'articles importants, Pierre-Yves Milcent donne, avec ce nouvel ouvrage, connaissance de son mémoire préparé en vue de l'obtention de son habilitation à diriger des recherches, brillamment soutenue en décembre 2010 à l'École normale supérieure à Paris. Il ne s'agit pas d'une simple édition à l'identique, mais bien d'une version remodelée et enrichie.

Une première remarque à propos du titre : si les élites sont omniprésentes, il s'agit, pour l'essentiel - ce que le sous-titre explicite - d'une sériation révisée en profondeur des dépôts de bronze non seulement de la Gaule atlantique, mais encore de l'ensemble de l'Arc atlantique, des îles Britanniques à l'Ibérie, pendant l'ensemble du Bronze final et le Premier âge du Fer ancien.

La rédaction de l'ouvrage est d'une qualité à laquelle nous ne sommes, malheureusement, que de moins en moins habitués. Le propos est clair, étayé par la mise en œuvre d'une érudition remarquable : P.-Y. Milcent n'ignore à peu près rien des publications anciennes comme récentes concernant le monde atlantique de l'âge du Bronze, il possède aussi une solide connaissance des données pour l'Allemagne, l'Europe centrale et l'Europe du Nord, qui lui autorise de fructueuses mises en perspective. 
4 Les points de vue défendus en début d'ouvrage balayent des idées reçues telles que le prétendu décalage culturel entre France de l'Est et de l'Ouest, le tropisme méditerranéen, les notions de centre et de périphérie, ou encore la définition de bien de prestige, etc. Souhaitons que ces mises au point bienvenues soient - enfin! intégrées par la réflexion de nos collègues!

5 Le terme proposé de " Gaule de l'Ouest », qui étonnera certains du fait qu'il est question de l'âge du Bronze, nous paraît tout à fait recevable, du fait des incontestables continuités de cette période à la période laténienne, continuités qui, pour certaines régions au moins, comme par exemple le Centre-Ouest, prennent leurs sources dès le Bronze moyen. P.-Y. Milcent démontre aussi que le rôle de l'Occident dans l'évolution culturelle ne doit pas être sous-estimé, comme il l'avait montré voici quelques années déjà dans un remarquable article consacré à la transition entre les deux âges du Fer (« Premier âge du Fer médio-atlantique et genèse multipolaire des cultures matérielles laténiennes ", in Celtes et Gaulois, l'Archéologie face à l'Histoire, 2: la préhistoire des Celtes, Glux-en-Glenne, 2006, p. 81-105). À ce sujet, P.-Y. Milcent propose, avec raison, une vision équilibrée qui évite de remplacer le désormais indéfendable tropisme oriental par un tropisme occidental, lequel évidemment serait tout aussi fallacieux, et propose la vision d'un monde multipolaire certainement autrement plus complexe que celui que les schémas traditionnels nous ont présenté.

6 Cet ouvrage si riche défie toute analyse détaillée dans le cadre de ce compte-rendu. La chronologie du Bronze final atlantique se trouve fortement précisée, chacune des étapes traditionnelles, dont la pertinence est reconnue, se trouvant divisée en deux phases, chacune désignée à partir d'un ensemble clos éponyme, un dépôt le plus souvent. Un fait notable, par exemple, est la confirmation de la date un peu tardive de l'horizon de Rosnoën - le BF I de J. Briard - dont il n'y a plus à douter qu'il est précédé par un « horizon de Malassis » pendant lequel dans les dépôts ou sur un habitat comme le Bois du Roc à Vilhonneur en Charente, des artefacts du BzD s'associent au fond traditionnel du Bronze moyen III, sans éléments annonçant l'horizon de Rosnoën (p. 83). Les réflexions sur le Premier Fer ancien ( $\mathrm{Ha} \mathrm{C}$ ) sont particulièrement novatrices.

7 Un problème non négligeable était d'éviter de réutiliser les termes BF I, BF II, etc., dont on sait de longue date que leur acception n'est pas la même selon qu'on traite de la chronologie occidentale ou de l'orientale. Pierre-Yves Milcent résout la question en ajoutant un a après $\mathrm{BF}$ : ainsi $\mathrm{BF}$ a1 désignera l'horizon de Rosnoën, etc. Espérons que cette sage pratique, que d'aucuns avaient sans succès déjà proposé antérieurement, sera cette fois suivie! Quant aux dates absolues des différentes phases du BF et du Premier Fer, elles posent des problèmes (insuffisance actuelle du nombre des datations $14 \mathrm{C}$ et quasi impossibilité des dates dendro-chronologiques en Occident) que PierreYves Milcent ne cherche pas à celer.

8 L'ouvrage démontre, n'en déplaise aux tenants d'une certaine mode actuelle, toute la nécessité de la poursuite d'une recherche archéométrique («jamais terminée ni jamais suffisante ", comme l'écrivait si justement le $\mathrm{Pr}^{\mathrm{r}} \mathrm{A}$. Möberg, un des grands noms de l'archéologie processuelle dans les années 70) seule à même d'apporter les données temporelles fines et fiables - ce que réussit pleinement P.-Y. Milcent, en proposant un phasage serré du Bronze final de la Gaule atlantique - indispensables préalables à toute tentative de reconstruction solide des sociétés du passé et de leur évolution sociale : la mise en évidence de phases d'abondance des dépôts et de phases de repli voire de disparition, en est une éclatante illustration. 
9 Une abondante iconographie, avec cartes, tableaux et graphiques, permet de suivre pas à pas les développements de l'auteur. Une riche bibliographie (p. 193-206), un inventaire des dépôts avec la proposition de précision de leur datation (p. 207-239) et un index toponymique suivi des résumés en français et en anglais complètent utilement l'ouvrage.

10 L'ouvrage de P.-Y. Milcent est incontestablement, pour la connaissance du Bronze final et du Premier âge du Fer ancien atlantiques, aussi important que le demeure depuis 1965 celui de J. Briard Les dépôts bretons et l'âge du Bronze atlantique. Il en constitue un remarquable prolongement, appelé à devenir à son tour et pour longtemps un socle indispensable pour les recherches futures.

\section{AUTEURS}

\section{JOSÉ GOMEZ DE SOTO}

Directeur de recherche émérite au CNRS, UMR 6566 « CReAAH », Rennes 